\title{
Une définition mixte de l'organisation : une intentionnalité théorique et méthodologique
}

\section{Olivia Belin}

\section{OpenEdition}

Journals

Édition électronique

URL : http://journals.openedition.org/communicationorganisation/196

DOI : 10.4000/communicationorganisation.196

ISSN : $1775-3546$

Éditeur

Presses universitaires de Bordeaux

\section{Édition imprimée}

Date de publication : 1 juillet 2007

Pagination : 180-195

ISSN : 1168-5549

\section{Référence électronique}

Olivia Belin, « Une définition mixte de l'organisation : une intentionnalité théorique et méthodologique », Communication et organisation [En ligne], 31 | 2007, mis en ligne le 01 juillet 2010, consulté le 21 décembre 2020. URL : http://journals.openedition.org/communicationorganisation/196 ; DOI : https:// doi.org/10.4000/communicationorganisation. 196 


\title{
Expériences
}

\section{Résumé}

Différents concepts et analyses peuvent être mobilisés pour traiter les problématiques organisationnelles d'introduction de technologies d'intelligence collective auprès de groupes d'utilisateurs. On peut notamment s'intéresser à l'organisation définie comme lieu d'exercice $d u$ pouvoir mais on peut également la comprendre comme lieu de communication et d'expression des structures culturelles, soit comme contexte. Ces définitions empruntent à divers courants théoriques de la sociologie des organisations, du Collège Invisible, et nous verrons aussi à la psychologie cognitive. Les analyses stratégique et contextuelle apparaissent complémentaires et facilitent la compréhension des phénomènes organisationnels complexes. De même orientent-elles l'intervention et l'action. Mais ces emprunts ne sont-ils pas aussi à intégrer comme une marque des méthodes constructivistes?

Mots clés: Technologies d'intelligence collective, pouvoir et communication, communication et culture

\begin{abstract}
Several concepts and analyses can be useful for treating organisational questions about the introducing of collective intelligence technologies among communities. The organisation can be both accepted as the place of powerness and as a specific place for communication, cultural expression, context. These definitions come from different theoretical schools of thought. Our strategic and contextual analysis will allow us, complementarily, to understand organisational and complex phenomenons for intervention and action. But, are these loans understandable either as a specificity of constructivist methods?
\end{abstract}

Keywords: Collective intelligence technologies, authority and communication, culture and communication

Olivia Belin est Attachée Temporaire d'Enseignement et de Recherche à l'Université de Montpellier 3 et rattachée au laboratoire du CERIC (le centre d'études et de recherche en information et communication). Ses domaines de recherche privilégiés sont «les méthodes qualitatives» (notamment la méthode systémique qualitative), «la recherche action», et «la communication des organisations ». Elle s'intéresse aux problématiques d'introduction des technologies de partage des connaissances dans les organisations. 


\section{Une définition mixte de l'organisation : une intentionnalité théorique et méthodologique Olivia Belin}

Olivia.Belin@univ-montp3.fr

Le chercheur en sciences de l'information et de la communication dispose d'une palette d'outils théoriques et méthodologiques pour recueillir ses données, construire et analyser ses objets de recherche, traduisant ainsi sa façon de penser et de traiter les problématiques qu'il souhaite aborder. La construction de l'objet de recherche peut se faire autour du concept même d'organisation, lieu de l'action collective organisée et lieu de communication.

Une définition « mixte » de l'organisation serait celle qui en rendrait compte, d'abord, comme le lieu privilégié de l'action collective organisée. On peut alors mobiliser la sociologie des organisations et s'appuyer sur le concept de pouvoir pour comprendre une situation d'introduction d'une technologie d'intelligence collective auprès d'un groupe d'utilisateurs. Mais l'organisation peut également être définie comme un lieu de communication, c'est-à-dire un "lieu d'activation des structures culturelles », comme le signalent les chercheurs du "Collège Invisible» où chacun agit en contexte. Emprunter alors le concept «d'affordance» à la psychologie de la perception et la cognition distribuée peut être judicieux pour comprendre les phénomènes de rejet d'un intranet.

Ainsi, les organisations pourraient être redéfinies comme des espaces de communication "par excellence », où projets collectifs et culture commune partagée (valeurs, normes, positionnement des acteurs) y sont comme exacerbés et disponibles pour des approches qualitatives. Les emprunts à divers courants disciplinaires, s'ils peuvent témoigner d'un effort de compréhension et d'action certain, ne sont-ils pas également la marque des approches constructivistes?

Nous prendrons appui dans ce texte sur le cas d'une entreprise déployant un intranet de partage et de communication. Dans une première partie, nous présenterons le cas sur lequel nous nous appuyons pour produire deux analyses stratégique et contextuelle. La première s'appuie sur le concept d'organisation comme lieu d'exercice du pouvoir, et la deuxième s'appuie sur l'organisation comme lieu de contextualisation et de définition des situations. Nous conclurons sur la complémentarité des deux approches, sur les intentionnalités méthodologiques et pratiques qu'elles traduisent et sur 


\section{Expériences}

le caractère éventuellement constructiviste dont cette recherche témoigne.

Contexte de la recherche et cas : un info-com au pays des sciences « dures »

\section{La communication : des pratiques et des théories}

Le terme 'communication' regroupe aussi bien des pratiques professionnelles que des théories. En contexte organisationnel, lorsque l'on parle de communication, on fait bien sur appel aux connaissances que chacun peut avoir sur la communication interne, externe et les pratiques en ces domaines. On s'intéressera alors aux plans de communication, aux relations presse, à l'image de l'entreprise... etc. En contexte scientifique et/ou universitaire, la communication renvoie à divers corpus théoriques servant à recueillir des données pour les analyser, sur des terrains relevant parfois des situations organisationnelles et des pratiques de communication.

Dans cet article, nous souhaitons témoigner du caractère migrant des analyses produites en SIC à partir d'une recherche menée lors d'une thèse et portant sur l'appropriation d'un intranet par une communauté de chercheurs. Il s'agit de traiter une problématique de terrain liée à la communication interne et managériale lors du lancement d'un intranet soutenu par un projet managérial fort visant la construction d'une communauté scientifique "forcée " par des logiques de partage accrues.

\section{Le contexte et le positionnement de l'enquêteur}

Notre recherche prend lieu et place dans un laboratoire d'informatique et est dirigée par un laboratoire SIC se réclamant des approches compréhensives. Une communauté de chercheurs en sciences dures constitue le terrain de recherche. Il s'agit d'amener les participants à un programme transversal et pluridisciplinaire, pilotés par un management fort, à utiliser un intranet de partage des connaissances initialement refusé. Des objectifs concrets de développement des usages et de perfectionnement de l'intranet de partage des connaissances font partie des attendus du laboratoire d'informatique qui développe l'intranet, comme du management de la communauté scientifique. Ces scientifiques sont tiraillés entre les mérites du site largement vantés par le management et les développeurs informaticiens alors qu'eux-mêmes ne font qu'en lister les inconvénients. Le positionnement de l'enquêteur est un positionnement qualitatif ou encore compréhensif, dans un double contexte scientifique des sciences exactes. Le laboratoire d'accueil est 
un laboratoire d'ingénierie informatique et le terrain est constitué de chimistes, physiciens, biologistes...

\section{La construction du cas : un premier choix parmi les éléments pertinents du contexte}

La situation que nous étudions peut-être abordée à partir du problème que poserait l'introduction de technologies de partage et de communication auprès d'une communauté scientifique, construite de manière directive autour d'une thématique fédératrice. Notre problématique de recherche, elle-même «processus de contextualisation $»^{1}$, se construit en prenant en compte les préoccupations d'un laboratoire d'informatique, celles d'un programme de recherche et de son management et celles des utilisateurs a priori démissionnaires (tel que le soulignent les managers du programme et les développeurs informatiques).

\section{Le site intranet du laboratoire d'informatique}

Le laboratoire d'informatique développe un logiciel de génération de sites internet et de gestion de contenu. Cet outil permet de développer, à partir de modules communs, des sites interactifs et dynamiques. Mais les utilisateurs ne sont pas au rendez-vous : pas d'alimentation autonome de données dans les bases, pas de participation aux forums, messagerie vide...

\section{Les enjeux et les moyens du management}

Pour le management, ce programme est l'occasion de se positionner sur des problématiques sociétales (le thème de recherche concerne la toxicologie nucléaire). Il permet également aux chercheurs de se valoriser à un niveau international. C'est également sur le plan managérial l'occasion d'une mise en œuvre transversale et pluridisciplinaire c'est-à-dire qui fait travailler différents laboratoires en mutualisation de leurs ressources. L'objectif implicite, selon le management, est de «décloisonner la recherche et de réussir à faire travailler, en interne, des laboratoires qui ont l'habitude de s'ignorer ». Le rapprochement des chercheurs (physique-chimie et biologie) et des recherches (fondamentale et appliquée) servirait ainsi les intérêts de chacun. La grande hétérogénéité marquant la communauté est parfois ressentie comme une richesse (on y trouve

\footnotetext{
1 Katambwe Jo M., "Recherche-action et consultation en communication des organisations : La production de savoirs sous double- contrainte " in colloque Recherche qualitative et production de savoirs, Université du Québec, 2004.
} 


\section{Expériences}

effectivement de nouvelles ressources en machines, savoir-faire et financement) mais également comme une menace (on y retrouve aussi ses concurrents). Enjeux politiques, sociétaux, managériaux et scientifiques se croisent.

Les chercheurs : excellence, réseau, confiance, partage restreint et hiérarchie

L'idéal d'excellence, explique un chercheur en biologie, est « de faire une grande découverte qui va bouleverser sa science, de trouver une molécule que personne n'a trouvée...». Cette excellence sera reconnue au travers des publications dans de grandes revues internationales. Les chimistes et les physiciens travaillent avec des «machines» et leur objectif est de mettre ces machines à disposition d'autres laboratoires (par exemple des laboratoires en biologie), dans des collaborations innovantes, dans des réseaux reconnus à un niveau international, d'excellence. L'obtention d'une position dominante se fait par la maîtrise de cette machine qui favorise la constitution d'un domaine de compétences où le savoir-faire, expliquent les chercheurs en physique ou chimie, représente un enjeu majeur, "une barrière à l'entrée ".

Les chercheurs en biologie se contraignent à des normes de secret, de confidentialité de la recherche ou de protection des résultats ${ }^{2}$ qui confinent parfois (pour des non-avertis) à la paranoïa. Cette logique touche les résultats du laboratoire, que ces résultats soient des résultats intermédiaires ou des résultats définitifs. Mais elle concerne aussi les protocoles de recherche. La loi du silence frappe toutes les informations qui circulent dans le laboratoire, lesquelles sont sous le contrôle du directeur de laboratoire, le seul à tout savoir vraiment. Les chimistes et physiciens, détenteurs de machines, ne donnent rien qui affaiblirait le positionnement du laboratoire. Les connaissances accumulées, par exemple, sur la manipulation d'une machine ne se divulguent qu' «au compte-gouttes» dans des collaborations restreintes. Et lorsque les chercheurs choisissent de collaborer, ils se mettent d'accord «le faire les yeux dans les yeux», la confiance que l'on se porte étant un élément déterminant dans la collaboration.

A noter que les rôles et places des acteurs sont définis et hiérarchisés de façon claire dans les laboratoires. Les chercheurs, doctorants et post-doctorants ont des rôles circonscrits à la production de la

2 Maurice Cassier, 1998, "Le partage des connaissances dans les réseaux scientifiques: l'invention des règles de bonne conduite par les chercheurs", Paris, Revue française de Sociologie, 4, pp 701-720 
recherche. Le directeur de laboratoire s'occupe des relations avec les partenaires, l'institution...

La connaissance comme enjeu communicationnel ${ }^{3}$

Il apparait que la connaissance scientifique et le savoir dont dispose chaque laboratoire n'ont pas de valeur du seul point de vue scientifique : ils sont également source de positionnement. C'est-àdire que le savoir sur les objets de recherche ou les outils et machines de recherche permet tout autant de poursuivre des travaux scientifiques que de développer des relations et des collaborations stratégiques. Ainsi, un chercheur témoigne : "Les machines, c'est une question de politique. Tout est politique ici $»$. Ainsi les connaissances s'utilisent comme pouvoir de négociation plaçant son détenteur dans une position avantageuse à mesure que les connaissances produites sont reconnues d'excellence. Plus une connaissance est reconnue, plus il est facile au laboratoire de négocier des collaborations, des financements, de la carrière, de la reconnaissance personnelle.

\section{L'organisation comme lieu d'exercice du pouvoir}

Un des concepts disponibles pour l'analyse organisationnelle est celui de pouvoir. Il permet de sonder une dimension relationnelle, et donc communicationnelle, de l'action collective.

\section{Comprendre à partir du concept de pouvoir la sociologie des organisations}

On peut mobiliser le concept de pouvoir et les problématiques s'y rapportant pour comprendre les phénomènes organisationnels. L'organisation semble être le lieu même d'exercice du pouvoir tel qu'elle est abordée en sociologie des organisations. Le pouvoir est d'abord ce qui permet de fixer officiellement certaines normes afin d'orienter et d'organiser l'action collective. Sans la fixation de règles, il ne peut y avoir d'action collective organisée. Mais plus qu'un attribut, il s'agit de considérer des relations de pouvoir où aucun acteur n'est « jamais totalement démuni face à l'autre ${ }^{4} »$.

Dans son acceptation la plus large, le pouvoir est «la faculté et la possibilité dont un ou plusieurs individus ou groupes d'individus disposent pour appliquer, faire accepter, faire exécuter ou imposer

\footnotetext{
${ }^{3}$ Mathilde Bourrier, Le nucléaire à l'épreuve de l'organisation, Paris, PUF, Le Travail Humain, 1999

${ }^{4}$ Michel Crozier, Erhard Friedberg, L'acteur et le système, Paris, Editions du Seuil, 1977, pp 60-61.
} 


\section{Expériences}

[...] des décisions [...] à un ou plusieurs individus ou groupes d'individus $[\ldots]^{5} \gg$. Henry Mintzberg ${ }^{6}$, par exemple, propose une compréhension du pouvoir comme l'influence d'un groupe d'acteurs sur les décisions stratégiques de l'entreprise, ces acteurs pouvant être des acteurs internes ou externes à l'entreprise.

Michel Crozier et Erhard Friedberg définissent le pouvoir dans l'organisation comme ceci : «Le pouvoir de $\mathrm{A}$ sur $\mathrm{B}$ correspond à la possibilité pour A d'obtenir que dans sa négociation avec $\mathrm{B}$, les termes de l'échange lui soient favorables ${ }^{7} \gg$. Dans cette acception de la notion de pouvoir, on met l'accent sur la négociation entre les acteurs, le pouvoir résidant dans la «marge de liberté dont disposent les acteurs $\|^{8}$. Cette définition rappelle celle de Max Weber, qui proposait de comprendre le pouvoir comme : « toute chance de faire triompher, au sein d'une relation sociale, sa propre volonté, même contre des résistances, peu importe sur quoi repose cette chance ${ }^{9} \gg$. Il existe différentes sources de pouvoir: l'expertise, la connaissance de l'environnement, la maîtrise des réseaux d'information, et la maîtrise des règles de l'organisation.

Aussi, pour ces auteurs, on ne peut comprendre le pouvoir en dehors de cette négociation au cœur de l'action collective, de telle sorte que le changement n'est pas seulement une décision : "Il ne s'agit pas de décider une nouvelle structure, une nouvelle technique, une nouvelle méthode, mais de lancer un processus de changement qui implique actions et réactions, négociations, et coopération. Il s'agit d'une opération qui met en jeu non pas la volonté d'un seul mais la capacité de groupes différents engagés dans un système complexe à coopérer autrement dans la même action ${ }^{10} »$.

Ainsi nous pouvons dire, dans notre cas, que le management cherche à contraindre les pratiques des scientifiques par un pouvoir institutionnel, d'une part, la maîtrise de la technique et de l'information d'autre part et enfin, l'expertise.

\footnotetext{
${ }^{5} \mathrm{http}: / /$ www.olats.org/schoffer/defpouv.htm , lien actif le 17/01/2007.

${ }^{6}$ Henry Mintzberg, Le pouvoir dans les organisations, Paris, Editions L'organisation, 1990.

${ }^{7}$ Michel Crozier, Erhard Friedberg, L'acteur et le système, Paris, Editions du Seuil, 1977, citant R.A. Dahl, « The concept of power », Behavioral Sciences, n², 1957, pp. 201-215.

${ }^{8}$ Crozier et Friedberg, L'acteur et le système, Paris, Editions du Seuil, 1992, p. 391, p. 69.

${ }^{9}$ Max Weber, Economie et société, Paris : Plon, 1971.

${ }^{10}$ Michel Crozier et Erhard Friedberg, L'acteur et le système, Paris, Editions du Seuil, 1992, p. 391
} 
Une définition mixte de l'organisation...

\section{Contraindre les pratiques des scientifiques}

Le pouvoir institutionnel

Dans notre cas, le site intranet repose sur une volonté managériale forte. Les managers mettent en œuvre à la fois un pouvoir institutionnel et un pouvoir d'expertise. Ils imposent un intranet du fait de leur position d'autorité. En quelque sorte, les scientifiques n'ont pas le choix : ils doivent utiliser l'intranet et les logiques de partage (où tout le monde a accès aux documents de tout le monde) ne sont pas discutables : elles sont imposées par la hiérarchie.

\section{Le pouvoir technique et de maîtrise de l'information}

D'autre part, l'objet technique est un argument renforçant un changement des pratiques professionnelles. Les managers imposent un intranet pour des changements organisationnels. Ce qui est visé, c'est un changement au cœur des pratiques de protection des données des scientifiques. Ils souhaitent ainsi amener les scientifiques à des formes de collaboration orientées vers le partage ou du moins, vers des formes de mutualisation des moyens. Aussi, sur cet intranet, l'ensemble des scientifiques ont accès à tous les documents de tous. En ce sens, ils cherchent à formaliser de nouvelles règles ${ }^{11}$ pour les acteurs de l'organisation en mobilisant dans la négociation des outils techniques qui conduisent, fixent et normalisent de nouvelles relations.

\section{Le pouvoir importé de l'expert}

Le site intranet est développé par un laboratoire d'informatique sous l'appellation d'une technologie d'intelligence collective ${ }^{12}$, système de gestion des connaissances. Les sciences cognitives, les sciences de l'ingénieur viennent souvent soutenir les discours officiels théoriques pour justifier d'un usage des outils de gestion des connaissances. De plus, toute demande de mise en place d'outils internet (forums, messagerie, stockage des documents) est systématiquement renvoyée auprès du laboratoire d'informatique, le seul habilité et capable d'intervenir sur de tels projets ${ }^{13}$. Or, ce laboratoire d'informatique

\footnotetext{
${ }^{11}$ Erhard Friedberg, Le pouvoir et la règle. Dynamique de l'action organisée, Paris, Editions du Seuil, 1993.

${ }^{12}$ L'intranet fait l'objet de nombreuses présentations lors de séminaires scientifiques, qui l'inscrivent dans divers courants de recherche des sciences cognitives.

${ }^{13}$ Dans le milieu du nucléaire (dont est issu le programme), les systèmes internet sont très fortement protégés.
} 


\section{Expériences}

soutient fermement le projet social et managérial de construction d'une communauté de partage au travers des outils d'intelligence collective. Il représente donc, pour le management, un pouvoir d'expertise importé de l'externe.

\section{Les marges de liberté des scientifiques}

Comment les scientifiques peuvent-ils répondre à cette injonction managériale de partage? Ont-ils la possibilité de contourner cette nouvelle exigence collective?

\section{Une attitude de fuite et d'évitement}

Les scientifiques impliqués dans le programme de recherche ne peuvent se soustraire totalement à l'autorité des managers. Aussi sontils obligés d'utiliser un minimum le site intranet et de participer aux réunions de formation et de réflexion sur les évolutions du site. Mais, en réalité, ils ne font que donner le change lors ces réunions et adoptent des stratégies d'évitement. En effet, beaucoup attendent la sortie de réunions pour dénoncer une "prise d'otage» ou encore invalider les objectifs de partage généralisé : «on ne fait pas trop comme ça de toute façon » alors qu'en réunion, ce problème de fond n'est jamais abordé. D'autres encore «évitent de donner des informations ", et beaucoup oublient l'adresse du site, leur code, leur mot de passe....

\section{Une maîtrise renouvelée et accrue des sources d'informations}

Les scientifiques renforcent également leur maîtrise des sources d'information. Les doctorants des laboratoires, post-doctorants, chercheurs non confirmés reçoivent de la part des directeurs de laboratoire des avertissements selon lesquels rien ne doit filtrer. Le laboratoire, plus que l'intranet, reste le lieu de production et de stockage des connaissances, connaissances dont les stratèges seuls peuvent disposer. Des informations «vieilles», «truquées voire fausses 》, "de faible importance » sont laissées à la communauté. Mais rien qui ne puisse remettre en cause les stratégies de positionnement entre laboratoires n'est laissé sur l'intranet.

\section{L'organisation comme contexte : quel débat latent autour du site intranet ?}

Le pouvoir est un vecteur de compréhension des relations dans les organisations, mais on peut aussi convoquer d'autres concepts tel que celui d'affordance, par exemple. 


\section{Comprendre à partir du concept d'affordance : le lien entre contexte et technique}

Le pouvoir est-il le seul vecteur de compréhension de l'organisation? Yves Fréderic Livian ${ }^{14}$ a déjà souligné par exemple que les deux leviers de l'action collective organisationnelle sont le pouvoir et la culture. Mais comment parler de cette culture ? On peut trouver dans les écrits des chercheurs de Palo Alto des éléments propres à la description de la culture en général et par extension à la culture d'entreprise. Communiquer, pour Birdwhistell par exemple, c'est «activer sa structure culturelle ${ }^{15} »$. C'est, nous dit encore Paul Watzlawick, "participer à une situation commune». Ainsi, les organisations sont traversées par des processus de communication, expression de la culture d'un groupe d'acteurs.

Comment cette culture s'exprime t-elle ? Ray Birdwhistell nous met encore sur la voie. Il étudie en kinésique la façon dont, en contexte, les significations passent au travers du corps. Il veut expliciter «le sens intentionnellement déposé dans le corps ${ }^{16} »$, le "corps n'étant pas découpé comme un mouton d'abattoir et une signification précise, indépendante de tout contexte et assigné à chaque morceau [...]. En d'autres termes, la signification flotte et ne se cristallise que dans un contexte défini.» L'homme malade de Winkin «joue » au malade, au "vrai et bon malade», pas au "geignard» ${ }^{17}$. La signification « mauvaise santé » est la mise en relation de multiples éléments, dans un contexte défini, commandant « une certaine présentation de soi».

La technique, dans les organisations, est une «réalité de premier ordre. [Elle] fait référence aux propriétés physiques des objets, la réalité de deuxième ordre renvoie aux propriétés sociales (valeur, signification de ces objets) ${ }^{18} \gg$. Elle participe donc à la construction progressive d'une situation commune partagée. On comprend alors l'importance d'un concept comme celui d'affordance issu de la psychologie de la perception ${ }^{19}$. Alex Mucchielli nous dit que «les

\footnotetext{
14 Yves Frédéric Livian, Introduction à l'Analyse des Organisations, Paris, Economica, 2000, 2e édition.

${ }^{15}$ Yves Winkin, La nouvelle communication. Recueil de textes, Paris, Editions du Seuil, nouv. édition 2000.

${ }^{16}$ Yves Winkin, La nouvelle communication, Paris, Editions du Seuil, 1981, p. 67

${ }^{17}$ Birdwhistell Ray L. (in Winkin, 81), Kinesci's and context. Essay on body motion communication, Philadelphia, University of Pennsylvania Press, 1970, p. 209.

18 Bateson, Birdwhistell, Goffman, et alii., La nouvelle communication, Textes recueillis et commentés par Yves Winkin, Paris, Seuil, 1981, p 59.

${ }^{19}$ Gibson, J. J. "The Theory of Affordances." In R. E. Shaw \& J. Bransford (eds.), Perceiving, Acting, and Knowing. Lawrence Erlbaum Associates, Hillsdale, NJ, 1977.
} 


\section{Expériences}

objets ne sont pas neutres. Ils sont porteurs de proposition d'interaction et donc «d'affordances » (c'est-à-dire de significations ${ }^{20}$ ) $\gg$. Pour Madeleine Akrich, l'avantage d'un tel concept est de prendre en considération simultanément l'acteur (souvent oublié dans les analyses sociotechniques), et l'objet dans l'action ${ }^{21}$. Et pour Thierry Bardini, on ne pourrait comprendre totalement ces affordances qu'en accédant «au monde virtuel des acteurs ${ }^{22}$ » et à leurs représentations. Les objets techniques et les dispositifs techniques renvoient donc à un ensemble de normes, de positionnement, de qualité des relations, de valeurs, lesquels forment un contexte dans lequel l'action se produit.

L'importation d'un tel concept permet de mener une analyse contextuelle intégrant simultanément les objets techniques (intégrés dans des dispositifs globaux) et la «culture» des acteurs. Il nous invite à explorer pratiques et logiques d'action sous-jacentes.

\section{Des dispositifs scientifiques et sociaux}

L'intranet est finalement, lui aussi, un révélateur des relations entre les acteurs, dans la mesure où il est inséré dans le dispositif scientifique et social des chercheurs. Il est associé à des pratiques et des logiques quotidiennes.

\section{Les pratiques et la place du site dans l'activité et les dispositifs quotidiens}

Le site intranet est marginalisé dans l'activité quotidienne des chercheurs: il n'appartient ni au dispositif de production de la recherche, ni au dispositif de valorisation de la recherche ${ }^{23}$. En matière de production, les pratiques sont en général fixées au travers de protocoles scrupuleusement respectés. En matière de valorisation, les scientifiques n'ont jamais besoin d'aller sur l'intranet. Ils ont déjà les publications qui concernent leur travail, ils reçoivent les informations nécessaires par d'autres moyens et le site ne fournit aucune indication sur les colloques, séminaires, revues... De plus, les documents administratifs qui constituent la majorité des documents de l'intranet s'adressent surtout aux directeurs de laboratoire.

\footnotetext{
${ }^{20}$ Mucchielli, Alex, La nouvelle communication, 2000, Paris, Editions Armand Colin, p. 173.

${ }^{21}$ Akrich Madeleine, Les objets techniques et leurs utilisateurs, de la conception à l'action, in Raisons Pratiques n4, Les objets dans l'action, pp. 35-57, 1993.

22 Bardini Thierry, Changement et réseaux techniques. De l'inscription aux affordances, revue Réseaux Nº76 CNET, 1996.

${ }^{23}$ Latour Bruno, La science en action, Paris, Editions La découverte, 1989.
} 
Les logiques globales et la valeur du site dans les laboratoires

L'intranet vient bousculer les logiques de secret et les positionnements hiérarchiques des laboratoires: il est en marge du contexte des acteurs. Les logiques de secret sont des logiques dépassant même les frontières des laboratoires et admises comme faisant partie des normes partagées par tous les chercheurs. Quant au positionnement entre chercheurs, il est mal accepté qu'un doctorant obtienne les mêmes informations que son directeur de recherche ou de laboratoire. Cela vient contredire la formalité des relations entre chercheurs. De plus, la proximité des scientifiques vis-à-vis du management est également une nouveauté, dans le sens où le chercheur a l'habitude "d'avoir les mains libres » entre « la nature et la société ${ }^{24} \gg$.

\section{Quand utiliser, c'est sortir du groupe}

Etre dans un laboratoire, c'est partager une réalité pratique, stratégique et sociale déterminée. Cela signifie "produire de l'excellence», "faire de bonnes collaborations", "protéger les informations $d u$ laboratoire $», ~ « n e$ rien dire aux concurrents $», \ldots$ Le site, tel qu'il se présente dans la situation de l'acteur, est un site qui propose un réajustement des enjeux scientifiques sur les enjeux managériaux, une déviance par rapport aux normes dans les laboratoires, et une activité subversive de partage et de divulgation de l'information. Utiliser le site, pour les scientifiques du programme, c'est sortir du groupe. C'est, en outre, initier de nouvelles pratiques scientifiques et changer de regard sur le monde de production de la recherche. Il s'agit pourtant, dans un projet autant scientifique, social et sociétal ${ }^{25}$, de questionner la responsabilité d'un chercheur engagé « au-delà de la science » dans des communautés ${ }^{26}$.

L'appropriation d'une technologie : un débat pour la redéfinition d'une situation collective partagée

Comment comprendre la volonté d'introduction d'un intranet dans l'organisation? Souvent les nouvelles technologies sont des

\footnotetext{
${ }^{24}$ Latour Bruno, La science en action, Paris, Editions La découverte, 1989, p. 349.

${ }^{25}$ On rappelle à ce titre que les enjeux scientifiques du programme sont d'apporter des réponses à la question de la toxicité des éléments radioactifs et que ces enjeux s'inscrivent dans une controverse sociétale majeure.

${ }^{26}$ Stengers, Isabelle, Le défi de la production de l'intelligence collective, entretien réalisé par Andrée Bergeron, www.multitudes.com, lien actif le 3/4/2007 et aussi «Les chercheurs font l'autruche».
} 


\section{Expériences}

révélateurs d'un débat interne latent ${ }^{27}$. Dans le cas exposé, le site intranet permet de rapprocher des acteurs qui d'ordinaire s'ignorent afin de redéfinir une situation collective partagée, un contexte. L'intranet pose la question de l'organisation d'une recherche débarrassée des effets pervers de la concurrence sur la structure et sur les laboratoires... mais concurrentielle quand même. L'intranet active un débat sur le contexte organisationnel. Ici, il n'est plus seulement le moyen par lequel le management entend asseoir ses décisions mais peut-être aussi une façon de repenser les modalités scientifiques ${ }^{28}$.

\section{La « migration » comme méthode constructiviste ?}

Mobiliser des concepts, cohérents sur le plan épistémologique et pour rendre compte des phénomènes organisationnels est-il un travail scientifique relevant seulement de l'engagement du chercheur ou peutil être aussi le propre d'une méthode constructiviste ?

\section{L'organisation comme lieu communicationnel par excellence}

L'organisation peut être considérée comme un lieu communicationnel par excellence, acceptant de multiples définitions. Les enjeux, les jeux de pouvoir, les cultures, les contextes y sont comme exacerbés. Le terrain et la richesse qu'elle constitue se livrent alors aisément aux différentes interprétations et méthodes d'analyse où le chercheur va « piocher » pour rendre compte pour lui-même et pour autrui d'un phénomène organisationnel particulier. Ainsi, on peut emprunter la voie des analyses stratégiques ou contextuelles, ces deux analyses acceptant l'import de concepts classiques, comme celui de pouvoir, ou de concepts plus récents comme celui d'affordance. Les deux analyses se complètent d'ailleurs sans se contredire et apportent chaque fois un éclairage différent sur une situation approchée en complexité.

\section{Une intentionnalité méthodologique, théorique et pratique ?}

Alex Mucchielli nous dit que «les connaissances élaborées [dans les méthodes qualitatives] doivent être : construites ; inachevées ; plausibles, convenantes et contingentes ; orientées par des finalités ; dépendantes des actions et des expériences faites par les sujets connaissants ; structurées par le processus de connaissance tout en le structurant aussi ; forgées dans et à travers l'interaction du sujet

\footnotetext{
${ }^{27}$ Morillon Laurent, Belin Olivia, «L’intranet comme révélateur des libertés : jeux, pouvoirs et stratégies d'acteurs dans les organisations », pp. 192-195, Colloque international «Pratiques et usages organisationnels des Sciences et Technologies de l'Information et de la Communication », Rennes, 7-9 septembre 2006.

${ }_{28}$ Isabelle Stengers, La nouvelle alliance, Métamorphose de la science, Paris, Gallimard, 1979.
} 
connaissant avec le monde ${ }^{29} \gg$. Notre cas illustre donc, par l'emprunt de concepts à différents courants disciplinaires, l'aspect constructiviste de la démarche de compréhension et d'analyse les phénomènes organisationnels.

On perçoit notamment la finalité instrumentale et scientifique des analyses stratégique et contextuelle. Elles permettent de produire une connaissance liée à l'introduction d'une TIC et d'agir dans l'organisation (le site sera finalement transformé à partir de la connaissance que l'on a du contexte des utilisateurs). On illustre bien la façon dont le sujet connaissant interagit avec le monde, scientifique d'une part, et organisationnel d'autre part. De plus, l'élaboration progressive des méthodes d'analyses (par le pouvoir? par les objets techniques? par les significations? par les affordances ?) témoigne d'un effort de recherche et du processus structurant et à structurer de la connaissance, permettant au chercheur de rendre accessible pour lui-même et pour les autres les objets auxquels il s'intéresse.

\section{Conclusion}

La «migration conceptuelle» permet donc aux chercheurs en information et communication de traiter des problématiques organisationnelles complexes et de le faire avec une certaine souplesse intellectuelle. Les deux approches qui ont été proposées ont un ancrage épistémologique proche, ce qui renforce la complémentarité des analyses. Mais le chercheur est parfois amené, dans sa démarche de compréhension, à investir des méthodes et des théories plus rationnelles, ne serait-ce que pour être en phase avec ses terrains d'intervention. On pourra s'opposer et s'interroger alors sur la valeur heuristique des emprunts conceptuels et sur "la sensibilité théorique ${ }^{30} \gg$ de celui qui mène ses recherches.

\section{Bibliographie}

Akrich Madeleine, «Les objets techniques et leurs utilisateurs, de la conception à l'action ", in Raisons Pratiques $\mathrm{n}^{\circ} 4$, Les objets dans l'action, p. 35-57, 1993.

Bardini Thierry, «Changement et réseaux techniques. De l'inscription aux affordances », Revue Réseaux N76 CNET, 1996.

\footnotetext{
${ }^{29}$ Alex Mucchielli, «Le développement des méthodes qualitatives et l'approche constructiviste des phénomènes humains ", Colloque de l'ARQ, Recherche qualitative et production des savoirs, hors série $\mathrm{n}^{\circ} 1,2004$.

30 Mucchielli Alex \& Al, Dictionnaire des méthodes qualitatives en sciences humaines, Broché, 2004.
} 
Expériences

Bourrier Mathilde, Le nucléaire à l'épreuve de l'organisation, Paris, PUF, Le Travail Humain, 1999.

Cassier Maurice, 1998, «Le partage des connaissances dans les réseaux scientifiques : l'invention des règles de bonne conduite par les chercheurs », Revue française de Sociologie, 4, p. 701-720.

Crozier Michel, Friedberg Erhard, L'acteur et le système, Paris, Editions du Seuil, 1977.

Dahl, R.A. "The concept of power », Behavioral Sciences, ${ }^{\circ} 2$, 1957, pp. 201-215.

Friedberg Erhard, Le pouvoir et la règle, Paris, Editions du Seuil, 1993.

Friedberg Erhard, L'analyse sociologique des organisations, Paris, Editions L'harmattan, 1987.

Gibson, J. J. "The Theory of Affordances ». In R. E. Shaw \& J. Bransford (eds.), Perceiving, Acting, and Knowing. Lawrence Erlbaum Associates, Hillsdale, NJ, 1977.

Katambwe Jo M., «Recherche-action et consultation en communication des organisations: La production de savoirs sous double- contrainte ", Colloque Recherche qualitative et production de savoirs, Université du Québec, 2004.

Latour Bruno, La science en action, Paris, Editions La Découverte, 1989.

Livian Yves Frédéric, Introduction à l'Analyse des Organisations, Paris, Economica, 2000, $2^{\mathrm{e}}$ édition.

Mintzberg Henry, Le pouvoir dans les organisations, Paris, Editions L'organisation, 1990.

Morillon Laurent, Belin Olivia, «L'intranet comme révélateur des libertés: jeux, pouvoirs et stratégies d'acteurs dans les organisations », pp. 192-195, Colloque international «Pratiques et usages organisationnels des Sciences et Technologies de l'Information et de la Communication », Rennes, 7-9 septembre 2006

Mucchielli Alex \& Al, Dictionnaire des méthodes qualitatives en sciences humaines, Editions Broché, 2004.

Mucchielli Alex, La nouvelle communication. Epistémologie des sciences de l'information-communication, Paris, Editions Armand Colin, 2000. 
Une définition mixte de l'organisation...

Mucchielli Alex, «Le développement des méthodes qualitatives et l'approche constructiviste des phénomènes humains, Colloque de l'ARQ ", Recherche qualitative et production des savoirs, hors série $\mathrm{n}^{\circ} 1,2004$.

Stengers, Isabelle, «Le défi de la production de l'intelligence collective ", entretien réalisé par Andrée Bergeron, www.multitudes.com, lien actif le 3/4/2007.

Stengers Isabelle, Prigogine Ilya, La nouvelle alliance, Métamorphose de la science, Paris, Editions Gallimard, 1979.

Weber Max, Economie et société, Paris, Edition du Plon, 1971.

Winkin Yves, La nouvelle communication. Recueil de textes, Paris, Editions du Seuil, nouv. Ed. 2000. 